\title{
El papel de las instituciones en contextos locales
}

\section{Kirsten Appendini}

El Colegio de México,

México, D.F.

kirsten@colmex.mx

\section{Monique Nuijten}

Wageningen University,

Países Bajos

mnuitjen@wxs.nl
En este artículo se examinan algunas cuestiones metodológicas que enfrenta la investigación sobre el desarrollo al estudiar las instituciones locales. Se estudia cómo formular conceptos operacionales útiles para captar información sobre las instituciones y sus procesos dinámicos en relación con las actividades económicas de los hogares rurales. Se presentan y evalúan algunos métodos cuantitativos y cualitativos sobre la base de estudios de casos en México y la India, así como sus alcances y limitaciones. Se concluye que se necesita un marco conceptual y metodológico multidisciplinario y flexible que permita entender las dinámicas de los procesos institucionales, desde las reglas y normas establecidas a las prácticas organizativas de la gente. Se subraya que la investigación relacionada con la formulación de políticas debe estar enfocada a objetivos específicos, como son los arreglos institucionales que pueden dar apoyo a los grupos más pobres y la manera como éstos pueden movilizarse para transformar las instituciones. 


\section{I}

\section{Introducción}

El tema de las instituciones ha estado en la mira del debate sobre el desarrollo durante varias décadas. Con la transformación del Estado y el reconocimiento de la existencia de "fallas de mercado" se ha hecho hincapié en las instituciones como el ámbito en el cual los agentes económicos y sociales tienen acceso a los recursos y pueden reforzar su potencial de ingresos. Así, "adecuar las instituciones" llega a ser el paradigma dominante en la formulación de políticas en la agenda del desarrollo internacional. Como consecuencia del ajuste estructural y de reformas económicas, las políticas intervencionistas dan paso a políticas enfocadas a la capacitación y devolución de poder a la población para hacer de ella socios activos en los esfuerzos de desarrollo. En este enfoque de "abajo hacia arriba", a las instituciones locales se les ha asignado un papel central en la tarea de apoyar a las personas frente al impacto de cambios macroeconómicos y de encontrar nuevas maneras de mejorar sus modos de vida a través del acceso a recursos y empleos. A nivel local, las instituciones se constituyen en instancias mediadoras que vinculan las políticas macro con los agentes económicos y sociales a nivel de la comunidad, así como en un marco en el cual, dadas las reformas estructurales, las personas se encuentran con "reglas del juego cambiantes", ya sea como individuos o colectivamente.
En este artículo queremos analizar algunas de las cuestiones metodológicas con las que se enfrenta la investigación sobre el desarrollo al examinar las instituciones locales. El estudio de las instituciones y la forma en que los individuos y los hogares rurales interactúan con el entorno institucional plantean importantes problemas metodológicos que han despertado poco interés en la literatura sobre el desarrollo. En el presente artículo se examina cómo se puede obtener información relevante sobre estos temas.

En primer lugar, se presenta un breve análisis de cómo se definen y utilizan los conceptos de institución y organización en la literatura sobre el desarrollo. Luego se discute cómo formular conceptos operacionales útiles para la investigación en el terreno. Finalmente, se presentan y se evalúan algunos métodos cuantitativos y cualitativos y las técnicas de investigación correspondientes, así como sus alcances y sus limitaciones para el estudio de las instituciones. La argumentación está ilustrada por varios estudios de casos en los cuales participamos y que se realizaron en México y la India. ${ }^{1}$ Pensamos que el análisis que se presenta puede ser de interés tanto para la investigación con fines académicos, incluidas aquéllas orientadas a la formulación de políticas o al diagnóstico con fines de proyecto y planificación, como para diversos agentes del desarrollo.

\section{II \\ El concepto de instituciones y organizaciones en el debate sobre el desarrollo}

Muchos proyectos de desarrollo atribuyen a las instituciones locales un papel central en el mejoramiento

\footnotetext{
${ }^{1}$ La investigación, que abarcó casos en India, México y Mozambique, se efectuó en virtud del Programa sobre Estrategias de Generación de Ingresos del Hogar para el alivio de la pobreza y las interacciones con el entorno institucional local, que llevó a cabo la División de Desarrollo Rural de la Organización de las Naciones Unidas para la Alimentación y la Agricultura (FAO). Participaron las siguientes instituciones: En India, Centre for Management in Agriculture, Indian Institute of Management (IIM), Ahmedabad. En México, Centro Re-
}

de las condiciones de vida de los pobres. Veamos en el recuadro 1 cómo subsiste cotidianamente una fami-

gional de Investigaciones Multidisciplinarias, Universidad Nacional Autónoma de México (CRIm/unam). En Mozambique, Departamento de Producción y Protección Vegetal, Facultad de Agronomía e Ingenería Forestal, Universidad Eduardo Mondlane, Maputo. Expresamos nuestra gratitud a todas estas instituciones; sin embargo, las opiniones expresadas son las de los autores y de su exclusiva responsabilidad. 
Recuadro 1

Vida de una Familia RURAL EN La INDia y EN MÉXICO

\section{India}

La aldea de Malawada en el distrito de Kheda, Estado de Gujarat, es una aldea de tamaño medio con una población de 3000 habitantes. Cuenta con un canal de riego durante todo el año. El arroz y el trigo son sus cultivos principales. Existe también una cooperativa lechera y una fábrica que emplea a algunos trabajadores de la aldea.

Jeevabhai y Ramabhai viven en la aldea de Malawada; pertenecen a la casta de los parmares. El hogar está formado por la pareja anciana y un hijo, su mujer y tres nietos. El hogar es propietario de cuatro acres de tierra heredada, de riego; también tiene un búfalo y dos becerros. Los adultos dividen su trabajo entre su propio predio y otros predios. Producen varios cultivos para los cuales compran insumos. También contratan mano de obra. Parte de la producción se vende a la cooperativa de la aldea. Jeevabhai es miembro de un grupo de crédito desde hace 15 años. Las mujeres pertenecen a un grupo de comercialización para vender la leche a una cooperativa lechera. El único requisito para ser miembro es tener animales productores de leche. Para participar en el grupo de crédito, Jeevabhai debe pagar una cuota. No hay que dedicar mucho tiempo para pertenecer a este grupo; los miembros dicen que han contribuido con uno o dos días de trabajo para cada organización y no tienen un papel activo en la toma de decisiones.

Fuente: IIM, 1999.

México

San Pablo está situado en los altos de la Sierra Juárez en el Estado sudoriental de Oaxaca. El bosque constituye el principal recurso natural de las comunidades y es de propiedad colectiva. La comunidad cuenta con un complejo marco institucional de gobierno que regula el acceso y la administración de los recursos.

Pedro, su mujer y una nieta viven en la aldea de San Pablo. Pedro es miembro de la comunidad y posee cuatro parcelas de tierra que fueron asignadas a su familia varias generaciones atrás. La familia cultiva maíz y algunos vegetales para consumo propio; tiene cuatro vacas. Pedro tiene derecho a trabajar en el bosque, lo que realiza contratando a un trabajador externo por día. De esta manera participa en la empresa forestal de la comunidad.

En 1964, Pedro era trabajador inmigrante en California. Actualmente, tiene tres hijas casadas que viven en Los Angeles, y un hijo que trabaja como mecánico y que manda regularmente dinero a su casa. La familia les ha prestado dinero para migrar. Son miembros de una asociación de migrantes. Otras dos hijas estudian en Ciudad de México.

Pedro ha desempeñado varios cargos dentro de instituciones locales, como el Comisariato (que administra los recursos comunes), y ha sido secretario, tesorero y síndico de la municipalidad. No recibió ningún sueldo por estas tareas, que forman parte de sus obligaciones como miembro de la comunidad.

Fuente: CRIM/UNAM/CRUCO/UACH (1999).

lia rural en la India y una en México y cuál es el papel de las instituciones.

Un breve vistazo al conjunto de actividades desempeñadas por estas dos familias y el contexto en que se desarrollan nos da una idea de la gran variedad de instituciones importantes para ellas (recuadro 2).

Como vemos, hay una gran variedad de elementos que caen bajo el término "instituciones". Por lo tanto, ante todo es preciso definir el concepto de institución y buscar la mejor manera de entenderlo en el contexto de una investigación específica, y luego decidir cómo estudiar las instituciones elegidas y su papel en la subsistencia de cada familia.
Con el fin de elaborar un marco conceptual para el estudio de las instituciones en contextos rurales se examinará ahora cómo se las ha enfocado en el debate sobre el desarrollo. Un rápido vistazo a la literatura más amplia muestra que existe confusión en la definición de los conceptos de institución y organización. Para empezar, ambos términos suelen intercambiarse. Además, distintas disciplinas y subdisciplinas, como la sociología de la organización, la nueva economía institucional, la sociología económica y la antropología, utilizan estos conceptos de maneras diferentes. Y por último, ni siquiera en el marco de una misma disciplina existe acuerdo en cuanto a su uso. 
Recuadro 2

INSTITUCIONES IMPORTANTES PARA LAS FAMILIAS RURALES, INDIA Y MÉXICO

El sistema de castas
La familia
El matrimonio
Los derechos de propiedad
La comunidad
Las reglas, leyes, constituciones
Los derechos de agua

El sistema de castas

La familia

El matrimonio

La comunidad

Los derechos de agua

\author{
El hogar \\ La asociación de migrantes \\ El sistema de cargos indígenas \\ El gobierno local \\ Los mercados de trabajo \\ Los mercados de insumos \\ y productos
}

\author{
El grupo de crédito \\ La empresa forestal \\ La cooperativa de la aldea \\ La cooperativa lechera \\ Las relaciones de género
}

Pese a la confusión y los desacuerdos, se puede distinguir cierta regularidad en la utilización de los términos. Cabe señalar que la mayoría de los estudios que intentan distinguir entre organizaciones e instituciones acentúan los aspectos normativos de las instituciones, mientras que hacen más hincapié en la parte estructural de las organizaciones. Por lo tanto, las organizaciones están más definidas en términos de estructuras de funciones reconocidas y aceptadas, mientras que las instituciones están más definidas en términos de creencias, normas y reglas que permiten el desarro1lo de estas funciones y estructuras (Uphoff, 1986; North, 1990; Ostrom, 1995; Scott, 1995). La distinción e interconexión entre organización e institución se puede ilustrar con el siguiente ejemplo. Las escuelas, la cooperativa lechera o la empresa forestal son organizaciones que existen porque una serie de 'reglas de trabajo' o instituciones subyacentes las definen y les dan un significado. Sin embargo, instituciones como el dinero, los mercados, el matrimonio y la ley no tienen una sola o directa manifestación organizacional (Leach, Mearns y Scoones, 1997, p. 237). En este artículo usamos el concepto institución en un sentido amplio que no solamente incluye aspectos normativos, sino también aspectos reguladores y cognoscitivos (Scott, 1995). La razón es que muchas instituciones (como los arreglos de tenencia de la tierra desarrollados a nivel local) son más bien prácticas rutinarias persistentes en el tiempo que un conjunto de reglas o normas (Leach, Mearns y Scoones, 1997, p. 91; Crowley y Appendini, 1998).

Muchos proyectos de desarrollo o investigaciones orientadas a la formulación de políticas argumentan que es importante clasificar las instituciones y organizaciones según ciertos criterios. Muchas veces se sostiene que las instituciones pueden ser clasificadas como formales o informales. Sin embargo, esta dicotomía no ayuda a captar el complejo de arreglos institucionales en el cual están involucrados los hogares. Otra distinción muy a menudo presente en la literatura es la que se hace entre las organizaciones de base de la comunidad, las organizaciones no gubernamentales y las agencias gubernamentales (Poulton y Harris, eds., 1988; Curtis, 1991; Bebbington y Ferrington, eds., 1993). Sin embargo, es difícil mantener estas diferencias en la práctica. Las organizaciones e instituciones son raramente privadas o públicas y muchas instituciones exitosas son una mezcla de instituciones de "tendencia privada" y de "tendencia pública" que desafía clasificaciones en una dicotomía estéril (Ostrom, 1995, p. 14). Otra clasificación común pero difícil de mantener es aquélla entre instituciones locales y extralocales. Muchas veces cuesta determinar lo que es o no es una institución local, y trazar límites estrictos parece ser un ejercicio irrelevante. En un contexto en el cual la gente opera cada vez más de un modo que trasciende límites e identidades territoriales específicas, tendríamos que encontrar nuevas maneras de definir 'lo local'. Sostenemos que el concepto local hace referencia a la incrustación (embedding) de realidades a mayor escala en mundos concretos (Appadurai, 1997, p. 55). Así, cuando hablamos del contexto institucional local nos referimos al entorno institucional relevante para los hogares en las áreas geográficas específicas que estamos estudiando.

Un problema presente en gran parte de la literatura sobre las instituciones y organizaciones locales para el alivio de la pobreza (Esman y Uphoff, 1984; Korten, 1987; Harris, 1988; Curtis 1991) es su referencia a acciones y objetivos colectivos. De hecho, la mayoría de los trabajos sobre "organizaciones o instituciones para el desarrollo" miran a las instituciones como unidades sociales dirigidas al logro de objetivos colectivos o a la satisfacción de necesidades institucio- 
nales de la sociedad o del entorno del cual son un componente. Sin embargo, el concepto de institución como estructura normativa y reguladora implica automáticamente la existencia de diferencias de poder e intereses divergentes entre la gente involucrada en ella. En nuestro trabajo vemos las instituciones como órdenes negociados que son construidos, mantenidos y transformados por la interacción social. Las instituciones están vinculadas a configuraciones de poder y dominación más amplias y pueden reproducir las obligaciones ideológicas y políticas a las cuales están atadas. Finalmente, debe reconocerse que las realidades institucionales y organizacionales son construidas, mantenidas y modificadas por procesos de creación y promulgación culturales. Por consiguiente, se crean valores y se expresan ideologías, rituales y ceremonias que llevan a un sentido de participación y pertenencia (véase Reed, 1992, pp. 73-129).

Para poder captar bien las diferentes formas de organización que son importantes para los hogares usamos también otro concepto: el de prácticas organizativas. Las prácticas organizativas hacen referencia a las distintas acciones y estrategias que sigue la gente para mantener y desarrollar su subsistencia cotidiana y otros proyectos de vida. Varios antropólogos sostienen que en lugar de empezar por la institución es más importante comenzar por las actividades de los individuos y de los hogares. Argumentan que la gente rural organiza sus actividades de distintas maneras en su vida cotidiana. Por tanto, muchas veces no se establecen organizaciones, pero se movilizan redes para proporcionar información crucial, apoyo financiero y ayuda práctica (Wolf, 1990; Long, 1990). Esto significa que habría que tomar nota de la existencia de las formas organizativas establecidas por los pobres antes de intentar influir en los ámbitos institucionales existentes. Esas prácticas organizativas tienen muchas veces un carácter fragmentario, no colectivo. Sin embargo, con el tiempo pueden devenir en formas de ordenación y regularización (Nuijten, 1998). Cuando con el tiempo estas prácticas de organización se estructuran más y se cambian ciertas reglas implícitas, podemos decir que se institucionalizan.
Por varias razones, argumentamos que es importante prestar atención a las prácticas organizativas para analizar las instituciones locales. Primero, las estructuras, que consisten más en prácticas rutinarias que en un conjunto de reglas o regulaciones establecidas, se encontrarán más fácilmente a partir de un estudio de las prácticas organizativas que mediante uno enfocado a las instituciones. En segundo lugar, un estudio de las prácticas organizativas puede profundizar nuestro conocimiento sobre la manera en que formas más estructuradas se desarrollan o no. También puede explicar por qué muchas veces prevalecen formas de organización más individualizadas y fragmentadas sin llevar a proyectos colectivos. Y puede sugerir recomendaciones de política con miras a la consolidación de ciertas modalidades informales de organización. En tercer lugar, un enfoque importante en los estudios de prácticas organizativas es el que considera los conflictos y las tensiones. Esto, en contraste con muchos estudios de instituciones que tienden a enfocar funciones u objetivos colectivos, o se apoyan en la suposición básica de que los conflictos se resolverán finalmente a través de la cooperación (por ejemplo, los neoinstitucionalistas). En cuarto lugar, los estudios de prácticas organizativas nos impedirán caer en la tendencia a reificar a las instituciones y atribuirles papeles que no desempeñan para la gente involucrada.

En consecuencia, se argumenta que si se combinan estudios enfocados a las instituciones y organizaciones con otros que hacen hincapié en las prácticas organizativas (empezando por las actividades de los actores sociales) se puede obtener una visión más aguda de las interrelaciones de las actividades económicas de los hogares con las instituciones locales. Más que un modelo, proponemos un marco conceptual (véase el recuadro 3). Con un marco tan flexible se puede tener una mejor idea del papel de las instituciones en la vida de la población rural y evitar dicotomías artificiales como las de formal/informal, interno/externo, local/extralocal, privado/público, moderno/tradicional. De esta manera, se toman en cuenta distintas manifestaciones de instituciones y resulta más fácil hacer frente a la heterogeneidad y a la complejidad. 
Recuadro 3

CONCEPTOS CENTRALES

\section{Instituciones}

Las instituciones son estructuras de tipo regulativo, normativo y cognoscitivo que dan estabilidad, coherencia y significado al comportamiento social. Las instituciones son transportadas por diferentes medios: cultura, estructuras y rutinas (Scott, 1995, p. 33).

\section{Organizaciones}

Las organizaciones son entidades construidas en torno a procesos definidos que aseguran el logro de objetivos determinados. La estructura organizacional se basa en la definición de roles y funciones distintas y las reglas organizativas deben asegurar un desempeño confiable (adaptación de Scott, 1995, pp. 48-49).

\section{Prácticas organizativas}

Las prácticas organizativas son las distintas acciones y estrategias de los individuos para sostener y desarrollar su subsistencia cotidiana y otros proyectos de vida. Las prácticas organizativas pueden evolucionar para conformar patrones establecidos (procesos de institucionalización) y de esta manera dar lugar a nuevas instituciones.

Contexto institucional local

El contexto institucional local está constituido por las manifestaciones específicas de las instituciones en el área geográfica en estudio, a pesar de que dichas instituciones pueden cruzar los límites físicos del área.

\section{III}

\section{Clasificación de las instituciones}

Una vez que el equipo de investigación tiene un panorama general del ambiente institucional es importante clasificar las instituciones y organizaciones según el objetivo del estudio. Obviamente, se las puede clasificar y subdividir de innumerables maneras. Si el estudio se enfoca a la generación de ingresos de los hogares es importante clasificarlas sobre la base del acceso a los recursos, de las relaciones de producción o de las actividades económicas.

Teniendo en cuenta las instituciones estudiadas, se puede llegar a la clasificación que figura en el recuadro 4.

El objetivo de la clasificación presentada no es establecer categorías exclusivas sino ayudar a desarrollar estrategias apropiadas para el estudio y análisis de las diversas instituciones. Claramente, distintos tipos de instituciones requieren distintos tipos de estudio y análisis. No se puede estudiar y analizar de la misma manera un sistema de castas y un sistema de tenencia de la tierra, ni estudiar un club de fútbol como se estudian arreglos de crédito. Así, por ejemplo, preguntas sobre año de fundación, objetivos principales, número de socios o servicios prestados a ellos sólo tienen sentido respecto de ciertas organizaciones y no de otras instituciones. Respecto a unidades administrativas gubernamentales no hace sentido hablar en términos de membresía.

Consideramos que las cuatro grandes categorías de la clasificación (divisiones socioculturales principales, relaciones de producción, gobierno local y organizaciones) deben tratarse como temas distintos en el análisis final.

Varias de las instituciones y organizaciones arriba mencionadas caen en más de una categoría. Por ejemplo, los grupos de crédito, los bancos de cooperativas, etc., se mencionan entre las organizaciones, pero también deben tomarse en cuenta en las relaciones de producción. El caso es que cumplen papeles diferentes y pueden ser estudiadas desde diversas perspectivas (como entidad organizacional o como parte de la estructura de crédito).

Es necesario considerar por separado las relaciones de producción (tenencia de la tierra, relaciones de crédito y de trabajo), ya que son instituciones complejas constituidas por muchos elementos distintos.

Asimismo, es importante tener una categoría especial para el gobierno local, que puede incluir agencias locales de entidades gubernamentales, así como 
Recuadro 4

CLASIFICACIÓN DE LAS INSTITUCIONES Y ORGANIZACIONES ESTUDIADAS

1. Principales divisiones socioculturales

- relaciones de género

- relaciones de castas

- alianzas religiosas

2. Relaciones de producción

- tenencia de la tierra (renta, medianería, propiedad)

- crédito (prestamistas, formas de interés, grupos de crédito)

- relaciones de trabajo (faena, ayuda mutua, venta de fuerza de trabajo a cambio de alimento)

3. Gobierno local

- ejido/comunidad

- barrios con comité de ancianos

- jefes/autoridades tradicionales

- comités gubernamentales locales

4. Organizaciones

- Vinculadas con la producción

Directamente

Banco India, cooperativa de distrito Grupo de producción de las mujeres

Sociedad cooperativa de productores de aceite

Cooperativa lechera

Unión de crédito, grupo de crédito

Grupo de ahorro de las mujeres

Grupo de ahorro para la casta prajapati

Asociación de productores

- De bienestar general

Puestos médicos

Asociación de curanderos tradicionales

Redes de migrantes, asociaciones de migrantes

Tiendas de bienes de consumo subsidiadas

Proyectos de desarrollo (dependiendo del tipo de proyecto)

- Empresas privadas

Empresas

Un banco comercial

Una empresa de turismo ecológico (Elephant Coast Company)

Empresa turística

- De carácter social/religioso/educativo

Clubes de fútbol

Grupos de mujeres
Grupos de jóvenes

Grupos parroquiales/religiosos
Indirectamente

Grupo de servicios de trabajo agrícola rural Comités de solidaridad

Org. no gubernamentales (ONG)

Grupos de mantenimiento de los grupos de agua grupos de representación elegidos, como los consejos de la comunidad. Estas instituciones estarán frecuentemente compuestas por una mezcla de elementos públicos y privados.

Un número considerable de organizaciones puede subclasificarse de distintas maneras. La incorporación de ciertos criterios permite categorizar las instituciones de la manera que más interese. Por ejemplo:
- si la institución incluye o excluye ciertas categorías de la población,

- si la institución está enfocada hacia los pobres,

- si la organización tiene potencial de desarrollo.

Antes de examinar los distintos métodos que pueden ser útiles para el estudio de las instituciones es importante prestar mayor atención a la relación entre las instituciones locales y las actividades productivas 
de los hogares rurales. Es preciso darse cuenta de que los hogares operan en múltiples instituciones a la vez y que es la combinación de instituciones lo que determina la demanda y el acceso a los recursos. ${ }^{2}$ Por ejemplo, el derecho a la propiedad dentro de una aldea sería de poco uso para el hogar si no estuviera combinado con otros arreglos institucionales. Como lo veremos más adelante, los procesos de inclusión o exclusión institucional están también determinados por las acciones combinadas de diferentes instituciones. Finalmente, las instituciones pueden superponerse y cumplir distintas funciones a la vez. Esta dinámica se aclara con los ejemplos que aparecen en los recuadros siguientes.

Recuadro 5

RECOMENDACIONES PARA EL ANÁLISIS DE LAS INSTITUCIONES LOCALES

Si el propósito del estudio es entender los vínculos entre las estrategias de ingreso de los hogares y las instituciones locales enfocadas hacia los procedimientos de inclusión y exclusión, con el objetivo de formular políticas, en el análisis de las instituciones locales se recomienda:

- No tratar de presentar una visión "completa" de las instituciones locales.

Es imposible cumplir con esta tarea, e intentarla sólo llevaría a un trabajo superficial y vago.

- No atenerse a clasificaciones artificiales.

La mayoría de las instituciones están compuestas por elementos distintos y combinan, por ejemplo, características de empresas públicas y privadas. Por eso, no se debe usar categorías exclusivas.

- Definir la relación que existe entre las instituciones y la diferenciación socioeconómica.

Por ejemplo, de qué manera difiere el acceso a la tierra entre los diferentes grupos socioeconómicos.

- Determinar cuáles instituciones pueden ser consideradas en las políticas de desarrollo.

Por ejemplo, porque dan apoyo a hogares pobres y tienen potencial para un desarrollo más extenso, o porque, por el contrario, representan un obstáculo para el desarrollo de ciertos hogares y quizás podrían ser cambiadas.

\section{Recuadro 6 \\ LOS HOGARES Y LA DINÁMICA DE MÚLTIPLES INSTITUCIONES}

En el caso de la familia mexicana, las actividades económicas y el acceso a los recursos están determinados por el hecho de ser miembro de la comunidad, con derechos y obligaciones. Esto significa que Pedro participa en instituciones de gobierno local y de administración de recursos. El hogar está involucrado en instituciones vinculadas a los mercados de trabajo y de productos, así como a las redes de apoyo a la migración. Pero las instituciones no están sólo relacionadas con actividades económicas y de gobierno local. Pertenecer a la comunidad tiene un significado más amplio: es esencial en la vida de una persona. Los derechos y obligaciones son flexibles porque los miembros de la comunidad están involucrados en una migración intensa y han creado mecanismos para no perder sus derechos, cumpliendo con sus obligaciones aun cuando se encuentran fuera de la comunidad. Por lo tanto, la respuesta a la pregunta de quién tiene cuáles derechos y obligaciones y cuándo los tiene puede no ser directa, y el acceso y uso de la tierra están mediados por otras instituciones y formas de organización (Appendini, García y De la Tejera, 2001).

En el caso de la familia india, varias instituciones son importantes para las actividades productivas: la posesión de la tierra, la administración del agua, el mercado de trabajo, el mercado de insumos y productos locales, la cooperativa lechera y los grupos de crédito. Ramabhai puede vender leche a la cooperativa porque, al poseer tierra, la familia puede dar forraje a sus animales. Jeevabhai pertenece a un grupo de crédito porque posee tierra, condición para ser socio. Aquí las instituciones también están vinculadas. Por ejemplo, la membresía en la cooperativa lechera está vinculada a la propiedad de tierra y a los acuerdos de mediería (conseguir forraje para los animales). Para el hijo de Jeevabhai y su familia el acceso a la tierra está conectado con las relaciones de parentesco y de matrimonio, ya que sólo lo tienen en su calidad de miembros de ese hogar.

\footnotetext{
${ }^{2}$ Véanse Leach, Mearns y Scoones (1997) y Crowley y Appendini (1998).
} 
Recuadro 7

RELACIONES ENTRE HOGARES RURALES E INSTITUCIONES

- Los hogares operan en múltiples instituciones al mismo tiempo.

- Es la combinación de distintas instituciones la que determina las demandas y el acceso a los recursos y la posibilidad de ciertas actividades productivas.

- Los procesos de inclusión y exclusión institucional son el resultado del funcionamiento combinado de distintas instituciones.

- Muchas veces las instituciones desempeñan distintas funciones a la vez: proporcionan insumos para las actividades productivas, proveen formas de seguridad social y brindan elementos que son centrales para la identidad de la gente y su sentido de la vida.

- La integración institucional difiere conforme al estrato socioeconómico. Los enlaces institucionales con que cuentan los grupos más pobres de la población rural difieren de aquéllos de sus grupos más ricos.

\section{IV}

\section{Una discusión metodológica para el estudio de las instituciones}

En esta sección analizaremos cómo aproximarse al estudio de las instituciones locales en la investigación de campo. Postulamos que es necesario tener un enfoque abierto y flexible que combine metodologías cualitativas y cuantitativas. Basándonos en una evaluación crítica de la experiencia con estudios de casos, nos referiremos principalmente a la utilización de encuestas y a metodologías cualitativas.

En los estudios de casos se tuvo como punto de entrada a las comunidades la utilización de técnicas de diagnóstico basadas en diseños muy en boga entre los organismos de desarrollo internacionales y en el diseño de programas y proyectos de organismos gubernamentales, como el diagnóstico rural participativo (DRP) y el diagnóstico rural rápido (DRR). El objetivo de estas técnicas y de tipos similares de diagnósticos es proveer información sobre los temas centrales de la investigación y del contexto etnográfico. En los estudios llevados a cabo en los tres países se utilizó el DRP/DRR para efectuar una evaluación general de la comunidad. Se obtuvo información sobre los recursos principales, las actividades económicas y la estratificación socioeconómica de los hogares, así como acerca de las instituciones más importantes.

Sobre la base de esos estudios se concluyó que el DRP no era una herramienta suficientemente poderosa como para determinar la relevancia de diferentes ins- tituciones. Ciertos instrumentos de DRP, como los diagramas de Venn, tienden a inclinarse por las organizaciones y excluyen instituciones menos formalizadas que a menudo son centrales para las estrategias de generación de ingresos de los hogares, como los distintos mecanismos para obtener crédito, las relaciones de trabajo recíprocas y los acuerdos para la posesión de tierra. $^{3}$

En esta primera fase de la investigación de campo resaltaron algunas experiencias que sirvieron como referentes a lo largo de todo el trabajo. Una es que en el proyecto la investigación no siempre puede realizarse de manera lineal, ya que los equipos de investigación necesitan avanzar y retroceder, y tener la posibilidad de adaptar los planes de investigación cuando lo parezca necesario.

Una de las mayores limitaciones de la investigación orientada a la formulación de políticas es el tiempo. Debido a que suele disponerse de poco tiempo para realizar la investigación de campo, muchas veces se sacrifica la recolección detallada de datos, lo que puede conducir a un diagnóstico erróneo y a malas

\footnotetext{
${ }^{3}$ Hay una interesante literatura evaluativa sobre la aplicación de estas técnicas. Véase Mosse (1994 y 1998); también Richards (1995), citado por Mosse (1998).
} 
recomendaciones de política. Aunque esto constituye siempre un dilema, se puede resolver hasta cierto punto con una investigación bien focalizada. Es preferible focalizar la investigación en un tema seleccionado que disponer de muchos datos poco detallados en un rango amplio de temas. Más que una perspectiva abierta, lo que se necesita es identificar claramente, y en una fase temprana de la investigación, los objetivos del estudio en términos de instituciones, intereses e intenciones de desarrollo. Esto es crucial para determinar la metodología que ha de adoptarse y el enfoque necesario para la investigación.

Por último, la experiencia y las calificaciones de los miembros del equipo de investigación influyen decisivamente en los resultados. En muchos países es difícil encontrar equipos de investigación altamente calificados y experimentados. De allí que la formación de tales equipos puede ser un objetivo de la propia investigación, y de una estrategia para la formación de recursos humanos — a nivel local/regional/nacionalque sustente las investigaciones participativas. Por esta razón, también es importante incorporar a gente local en el equipo y apoyar la formación de recursos humanos en las comunidades.

A continuación queremos ver cómo determinar ciertas características de las instituciones y el papel que desempeñan en las estrategias económicas de los hogares usando datos cuantitativos obtenidos de encuestas.

Mediante una encuesta de hogares se recopilaron datos cuantitativos sobre las instituciones vinculadas a las actividades generadoras de ingresos, con el fin de apoyar el material sobre las interrelaciones institucionales obtenido a través de los diagnósticos comunitarios, algunas entrevistas, estudios de casos, etc. ${ }^{4} \mathrm{El}$ que sea necesario o no recoger información mediante cuestionarios y, en caso positivo, el tipo de información que se requiere depende de las instituciones específicas que haya que estudiar.

Los datos obtenidos por medio de encuestas de hogares pueden ser útiles para identificar las institucio- nes que tienen mayor importancia para los distintos grupos sociales de la comunidad. Por ejemplo, en el contexto de las relaciones de tenencia de tierra habría que reunir información sobre el número de hogares que cultivan la tierra con distintos tipos de contratos de tenencia y sobre la superficie cultivada en una misma comunidad bajo cada tipo de contrato. En el contexto de una organización, digamos un grupo de crédito, se debería recopilar información sobre el número exacto e identidad de los hogares que participan en ella. En la mayoría de los contextos esa participación se puede estudiar de diversas maneras. En el caso de un grupo de crédito puede analizarse, por ejemplo, en términos de membresía en el grupo y de crédito adelantado por el grupo a diversos hogares. Esta información tendría que ser revisada teniendo en cuenta la encuesta de hogares y las entrevistas cualitativas. En Malan, India, todos los hogares tienen acceso formal a la cooperativa lechera, pero sólo aquéllos con acceso a la tierra y al forraje pueden mantener ganado y así producir leche.

Las encuestas de hogares permiten recoger información de amplio espectro sobre arreglos y prácticas institucionales. También pueden incluir otros aspectos relativos a la participación, como la información sobre las reglas, los derechos y las obligaciones (recuadro 8$).^{5}$

En el caso de México se puede concluir que existen prácticas diferentes en las comunidades, que dependen de la forma en que se toman decisiones acerca de las parcelas abandonadas. En San Pablo, donde el ganado constituye una fuente de ingreso importante, las parcelas agrícolas no se pierden si están cercadas para pastoreo (privatización); en Ixtlán, algunas parcelas pasan a ser parte del bosque para la explotación comercial (colectivización). Sin embargo, las distintas prácticas organizativas no siempre se ajustan de manera suave a los cambios que se producen con el tiempo, de modo que los conflictos y la erosión de las instituciones pueden ser parte de estos procesos. Para investigarlo, recurrimos a información cualitativa.

\footnotetext{
${ }^{4}$ La información sobre la vinculación de los hogares con las instituciones se recabó como parte de una encuesta amplia sobre las estrategias de los hogares para la generación de ingreso.
}

\footnotetext{
5 Por supuesto, existen también otros aspectos de la participación - por ejemplo, la participación en la toma de decisiones- que son cualitativos y deben estudiarse de manera diferente.
} 
Recuadro 8

MéXico: Preguntas sobre DeRechos, obligaciones y SANCIONES CON RESPeCto a LOS RECURSOS

La comunidad indígena constituye una institución envolvente que comprende los derechos a los recursos y el manejo de ellos por parte de sus miembros, así como la participación en los órganos de gobierno local y las obligaciones y sanciones al respecto.

En la encuesta de hogares se preguntó sobre las reglas y normas de acceso a los recursos y uso de ellos, así como sobre las obligaciones y sanciones.

\section{Cuestionario}

Acceso a los recursos y derechos:

1. Al convertirse en un miembro de la comunidad, ¿se le asigna a usted individualmente a una parcela de tierra para cultivar?

- Superficie de la parcela

2. ¿Recibe usted el derecho a explotar áreas comunes?

- ¿De qué tipo? (tierra, tierra de cultivo común, bosque)

3. Como miembro de la comunidad, ¿tiene usted derecho a participar y a votar en la asamblea?

4. ¿Recibe usted el derecho a ocupar un cargo?

5. ¿Cuál cargo y en qué orden de importancia?

Acceso a los recursos y las obligaciones:

1. ¿Debe usted pagar alguna contribución a la comunidad? ¿Para qué? ¿Cuánto?

2. ¿Debe usted contribuir con alguna actividad laboral para la comunidad? ¿Con qué propósito? ¿En qué cantidad? Fecha de contribución

3. ¿Qué ocurre si usted deja su parcela sin cultivar?

4. ¿Es su presencia obligatoria en las asambleas? ¿Cuántas veces se celebraron asambleas el año pasado? ¿Para qué razones se organizaron esas asambleas?

5. ¿Participó usted en las asambleas? ¿Cuántas veces?

6. ¿Se ve usted obligado a cumplir con un cargo? ¿Cuándo?

7. ¿Existen otras obligaciones? ¿Cuáles?

Sanciones:

1. En el caso de que usted no cumpla con sus obligaciones como miembro de la comunidad, ¿se le somete a sanciones? ¿Cuáles?

2. ¿Son idénticas las sanciones para todo tipo de transgresión?

3. ¿Son idénticas las sanciones la primera vez que se comete una transgresión y cuando ésta se comete varias veces?

4. Si existen reglas para el acceso y uso del bosque (para el pastoreo/para el cultivo), ¿cuál es el grado de cumplimiento con estas reglas?

5. ¿Dónde se decidieron las sanciones (asamblea general; autoridades ejidales; el consejo de los ancianos; otro consejo; cualquier otro lugar (especificar)?

6. ¿Quién estuvo encargado de aplicar las sanciones?

7. ¿Hubo algún desacuerdo sobre las sanciones? Si lo hubo, ¿cómo se resolvió?

Algunas reglas de la comunidad relacionadas con los derechos a los recursos se han aflojado en la práctica o simplemente no están bien definidas. Esto sobre todo debido a la migración y al hecho de que los migrantes intentan no perder sus derechos por estar ausentes de la comunidad. Un ejemplo son los derechos a disponer de las tierras de cultivo que han sido abandonadas. Conforme a la ley agraria mexicana, el hogar pierde el derecho de cosechar la tierra si ésta deja de ser cultivada. Pero como respuesta a la pregunta de cuáles serían las consecuencias si una parcela no se cultivara, el 69\% de los hogares en las comunidades estudiadas contestó 'no pasaría nada'; el 17\% contestó 'se perdería la parcela y ésta sería asignada a otro miembro de la comunidad'; otras respuestas fueron 'una parcela abandonada durante cinco años o más se pierde'; 'no pasa nada si uno cumple con un cargo'; 'no pasa nada si la parcela está cercada' (San Pablo); 'si se plantan árboles en la parcela, ésta pasa a ser parte de las tierras forestales de la comunidad’ (Ixtlán).

Fuente: CRIM/UNAMO/ CRUCO/UABCH, 1999. 


\section{V}

\section{Métodos cualitativos para el estudio de las instituciones}

Como se explicó más atrás, una parte importante de los vínculos entre las estrategias de subsistencia del hogar y las instituciones locales no se podrá entender sólo con un estudio de diagnóstico o mediante una encuesta de hogares. Es preciso realizar otros estudios. Para captar las prácticas organizativas y la complejidad de las vinculaciones del entorno institucional superpuestas en los hogares rurales se necesitan métodos cualitativos de investigación (Mikkelsen, 1995). Como la investigación cualitativa toma mucho tiempo, son pocas las instituciones que pueden estudiarse de esta manera. En cada situación hay que decidir cuáles son las instituciones que por su importancia habrá que estudiar, y para cada una de ellas hay que desarrollar un plan de estudio especial. Por ejemplo, uno puede decidir estudiar las instituciones cuya importancia en las actividades de ciertas categorías de hogares ha sido demostrada (acuerdos sobre intercambios laborales, una cooperativa). También puede ser útil estudiar en detalle los procesos que llevan a excluir a ciertos grupos de la población de una organización de producción importante. De esta forma se puede intentar influir posteriormente en estos procesos de exclusión, por medio de un proyecto orientado a la formulación de políticas. En muchas situaciones será muy importante estudiar los conflictos y las tensiones subyacentes en organizaciones locales o en torno a acuerdos sobre la tenencia de la tierra, antes de introducir proyectos orientados a la resolución de conflictos o nuevas formas de gestión.

\section{Algunos métodos cualitativos}

Existe una literatura muy vasta sobre los métodos de investigación cualitativa (Strauss, 1987; Silverman, 1993; Alaasutari, 1995). Los siguientes métodos son especialmente apropiados para el estudio de las instituciones.

\section{a) Historias de vida}

En general, las historias de vida constituyen un método eficiente para revelar los esfuerzos e intentos de la gente para aliviar la pobreza. También pueden ser importantes para el estudio específico de los contextos institucionales en que están inmersos la gente y los hogares. Las historias de vida enseñan cómo los miembros del hogar intentan ganarse la vida, procuran satisfacer sus diferentes necesidades, hacen frente a ciertas incertidumbres, y reaccionan ante nuevas oportunidades y situaciones trazando diferentes redes sociales y nuevas formas de organización. En el estudio de las historias de vida, uno puede usar las genealogías como un instrumento metodológico adicional. Esto puede ser especialmente útil en áreas caracterizadas por la migración y el movimiento de la gente, o en áreas donde las redes migratorias son esenciales para la economía del hogar. Las series de trayectorias de vida de individuos y hogares, en combinación con el estudio de flujo de recursos, pueden ayudar a entender la relación entre procesos de movilidad social y redes de apoyo.

b) Relatos de historias y análisis de narrativas

Los relatos de historias están reconocidos como un elemento central del proceso de organización. Por esta razón, el análisis de relatos y narrativas es un método cada vez más utilizado en el análisis de las organizaciones. Pero el prestar atención a las historias personales de la gente es también importante por otras razones. La visión de los miembros del hogar acerca de su propia situación y la manera en que la gente reflexiona sobre sí misma y el mundo a su alrededor, nos indica algo sobre el contexto social y cultural en que viven y puede dar indicios sobre las divisiones sociopolíticas y las formas de inclusión y exclusión relacionadas con las instituciones. Esto es especialmente claro en el estudio de narrativas colectivas en las que más que las experiencias individuales se expresan las experiencias de un grupo social.

\section{c) Análisis de estudio de caso extendido}

En el método de estudio de caso extendido (Mitchell, 1983; Walton, 1992) se elige un objeto de estudio (acontecimiento o situación) que se examina en gran detalle: por ejemplo, la elección del comité ejecutivo de una organización o la implementación de un 
nuevo proyecto de gobierno. El estudio de caso trata de proporcionar mayores detalles sobre las interacciones y confrontaciones sociales, para dilucidar los procesos institucionales. Al prestar atención a los distintos grupos de gente involucrados, así como a los diferentes intereses en juego, las relaciones de poder y el contexto histórico se hacen centrales. De esta manera, los estudios de caso extendidos pueden revelar dinámicas de organizaciones no observadas al examinar la estructura formal de las organizaciones.

\section{d) Análisis de conflictos y tensiones}

Los temas polémicos, las áreas de tensión y los conflictos abiertos tienen importancia capital para cualquier análisis de las instituciones. Las situaciones conflictivas arrojan luz sobre los principales recursos en juego y las luchas de poder en torno a ellos. El estudio de conflictos revela de qué manera se organizan los actores sociales mismos, cómo se forman y se separan los grupos y qué es importante para diferentes categorías de hogares. También muestra cómo el funcionamiento de las instituciones está influenciado por las relaciones de poder, y cómo las reglas, leyes y procedimientos oficiales pueden convertirse en instrumentos políticos y ser utilizados como recursos legales por individuos y grupos para lograr sus objetivos en momentos y lugares particulares (Starr y Collier, 1989, p. 2).

\section{El uso de los métodos cualitativos: ejemplos en México y la India}

El equipo mexicano asignó un papel central a los métodos de investigación cualitativa, pues argumentó que para analizar la vinculación de los hogares con las instituciones locales era necesario conocer el contexto histórico de las comunidades y la dinámica de sus cambios económicos e institucionales.

La investigación tuvo como principio fundamental que la vida de los campesinos está basada en valores y normas históricamente desarrollados que influyen en las preferencias, el conocimiento y las expectativas acerca de distintas actividades de producción, como la agricultura, la cría de ganado y el comercio. A fin de comprender más a fondo cómo se conformaron las estrategias económicas de los hogares en un entorno institucional dinámico se hicieron historias de vida en hogares seleccionados.

A partir de los resultados de la encuesta se prepararon perfiles de las economías de los hogares. Sobre la base de estos perfiles y de la relación estableci- da con ciertas personas en las primeras fases de la investigación, se hizo una selección de familias en cada comunidad para la construcción de historias de vida. Se realizaron entrevistas abiertas para reconstruir la evolución del perfil del hogar en los últimos 30 a 40 años o incluso más para algunas familias. Las entrevistas abiertas estuvieron dirigidas a cubrir las actividades económicas de los hogares y los vínculos con las instituciones.

Se usó un enfoque interpretativo, esto es, se prestó atención a los significados atribuidos por los mismos campesinos a la realidad que les rodea. Estas realidades subjetivas son fundamentales para entender el desarrollo de las instituciones locales y las formas de cooperación existentes. También dan una indicación de la viabilidad de nuevas formas institucionales. La construcción de historias de vida se llevó a cabo de manera crítica y creativa, con el fin de llenar vacíos y aclarar malas interpretaciones o incongruencias, y se pidió a los campesinos que reflexionaran sobre esto. De este modo se crearon condiciones para que la gente se expresara con toda libertad y se estableció un diálogo reflexivo en el cual fue posible cuestionar historias contadas en momentos cruciales.

La información cualitativa permitió entender distintos aspectos de la dinámica de la toma de decisiones y el manejo de recursos, entre otros, de los recursos colectivos forestales que tienen importancia crucial en la vida comunitaria y de los hogares. A título ilustrativo, en el recuadro 9 se analiza un conflicto en la comunidad de San Pablo, en México, que tuvo impacto en la comunidad y que incluso obligó a que un grupo de pobladores emigrara.

La investigación realizada en San Pablo mostró que existen contradicciones y tensiones serias en los objetivos formales de la institución y la relación con los hogares. Las historias de vida, entrevistas abiertas y pláticas informales con la gente acerca de las empresas mostraron claramente que los diferentes hogares que participan en empresas forestales comunitarias tienen visiones diferentes acerca de los objetivos de tales empresas y de sus propios intereses principales en el proyecto. Centrándose en las prácticas organizativas, se estudió cómo se llevan a cabo diariamente las diferentes actividades de la empresa forestal comunitaria. Esto permitió conocer mejor los procesos organizativos y las posibilidades de cambio. Finalmente, los investigadores enfocaron explícitamente las tensiones, los problemas y los conflictos surgidos, y se realizó un análisis de conflictos. 
Recuadro 9

ANÁLISIS DE CONFLICTOS INSTITUCIONALES Y PROBLEMAS ADMINISTRATIVOS: una empresa forestal comunitaria (San Pablo, México)

En San Pablo se estableció en 1982 una empresa forestal comunitaria independiente. La comunidad tiene además un aserradero. ${ }^{a}$ Como en las demás comunidades forestales de la Sierra Juárez, el control del bosque fue entregado legalmente a las comunidades, después de arduas luchas para terminar con las concesiones que el Estado mexicano había otorgado a empresas privadas.

Formalmente, en las empresas forestales la comunidad debe articular tres objetivos: la reproducción de la familia, los intereses de la comunidad y la viabilidad comercial de la empresa forestal.

Se cumple el primer objetivo si la empresa es capaz de generar suficientes oportunidades de empleo con una remuneración aceptable para los trabajadores y los propietarios (los mismos comuneros). El segundo objetivo exige beneficios para la empresa que serán utilizados adecuadamente en favor de toda la comunidad (construcción de obras públicas, ayuda para las escuelas, pensiones para viudas y ancianos). El tercer objetivo requiere el desarrollo de una empresa que traiga consigo democracia, eficiencia y sustentabilidad.

La investigación mostró que hay incompatibilidad entre las necesidades técnicas/organizacionales de las actividades forestales y las actividades cotidianas de los hogares campesinos. Las festividades, los patrones de migración y la agricultura del maíz imponen limitaciones severas al funcionamiento de la empresa forestal. Por ejemplo, es muy difícil establecer rutinas de trabajo estables, organizar la supervisión de los trabajadores y garantizar el uso completo de la capacidad productiva de la empresa a lo largo del año.

Existe una tensión entre la acumulación de capital para el mantenimiento de la empresa forestal y la reinversión en ella, por un lado, y el gasto de los beneficios en obras públicas, festividades religiosas y redistribución entre los comuneros, por otro. Algunos comuneros prefieren la redistribución directa del beneficio en lugar de la formación de un fondo común para reinvertir en la empresa forestal. Los comuneros también pueden preferir una inversión en infraestructura social o programas de seguridad social en lugar de una reinversión forestal. Por otra parte, la falta de inversión adecuada puede reducir la competitividad de la empresa y el beneficio a largo plazo, y puede hacer imposible que ésta sea rentable. Esta tensión ha creado conflictos importantes en los trabajos forestales y fricciones políticas durante las asambleas.

Aunque en general se argumenta que los conflictos internos en las comunidades son un obstáculo para el desarrollo de instituciones eficaces, la investigación muestra que ellos también pueden hacer que surjan ideas y puntos de vista nuevos y dar pie a una competencia entre los individuos y los grupos que tal vez acelere los procesos de cambio y de adaptación institucional.

La investigación reveló una crisis profunda en la comunidad en torno a la empresa forestal, debido a un fraude del presidente del Comisariato de Bienes Comunales en 1988-1989 y el consiguiente conflicto interno entre los comuneros. Esto llevó a un largo proceso judicial y a numerosas expulsiones de la comunidad. Un nuevo grupo se instaló, pero persiste una pugna fundamental por el control político de los recursos forestales. Las autoridades usaron los ingresos de las empresas forestales para luchar contra los grupos rivales. Los conflictos son tan profundos que la vida social se ve seriamente amenazada en todos sus aspectos. Los comuneros se reúnen cada vez menos. La gente tiene cada vez más la obligación de acudir y se recurre a un sistema de castigo para los que no asisten. La búsqueda de consenso también disminuyó. Estos conflictos causaron rupturas en la comunidad, con secuelas de incertidumbre acerca de la administración futura de la empresa. Sin embargo, la mayoría de los comuneros sigue apoyando a la empresa forestal.

Fuente: CRIM/UNAM/CRUCO/UACH, 1999.

a La empresa forestal comunitaria está administrada por un comité operativo, un director y otros profesionales. La empresa tiene que rendir cuenta a la Asamblea de Comuneros (oficialmente la mayor autoridad) y al Comisariato de Bienes Comunales.

En la investigación en la India se efectuó un estudio de caso sobre la cooperativa lechera en la comunidad de Malan. El objetivo del estudio fue el de conocer más a fondo los procesos de participación y mecanismos de exclusión de los hogares en la cooperativa, tanto para la entrega de leche como para la adopción de decisiones administrativas (recuadros 10 y 11).

El estudio de caso de la India señala que la conexión entre la tierra, el acceso a forraje, la posesión de ganado, las castas y la participación en la cooperativa 
Recuadro 10

ANÁLISIS CUALITATIVO DE LOS PROCESOS DE INCLUSIÓN/EXCLUSIÓN (Cooperativa lechera de Malan, India)

En la investigación :

- hubo pláticas informales con la gente sobre temas relacionados con la cooperativa

- $\quad$ se entrevistó a miembros y no miembros de ella

- se puso cuidado en entrevistar a hogares de distintas castas y estratos socioeconómicos

- se entrevistó a los gerentes de la cooperativa

- se preguntó a la gente por sus razones para ser miembros o no miembros

- se preguntó acerca de las razones por las cuales hay distintas formas de participación o de no participación

- se preguntó sobre el funcionamiento de la cooperativa, la producción, los servicios prestados, la administración y la toma de decisiones

- se estudiaron los conflictos y tensiones que fueron percibidos

- se asistió a las reuniones de la cooperativa

- $\quad$ se dio seguimiento a las actividades diarias de las personas

Fuente: Rawal, 1999.

Recuadro 11

ANÁLISIS DE UN ESTUDIO DE CASO

(Cooperativa lechera de Malan, India)

La cooperativa lechera Malan Milk Producer's Co-operative Dairy, en la aldea de Malan, en el estado de Gujarat, tiene como actividades la comercialización de leche, la venta de forraje para el ganado y la venta de ghee (un producto parecido a la margarina). La cooperativa funciona también como aval de los miembros que obtienen créditos para comprar ganado. Asimismo, presta servicios de veterinaria en la aldea. Para ser miembro de la cooperativa hay que poseer ganado y entregar leche regularmente por un mínimo de 90 días. Para ello la cooperativa cuenta con tres centros de acopio en la aldea.

La investigación mostró que la participación difería marcadamente según la casta, siendo mucho mayor la participación de los hogares pertenecientes a las castas superiores Hindu que la de los hogares pertenecientes a castas de la jerarquía más baja (scheduled).

El estudio identificó algunas de las barreras a la participación en la cooperativa. En primer lugar, siendo Malan una zona agroecológica de secano, la condición principal para poseer ganado era tener acceso a forraje. El acceso a forraje resultó estar estrechamente relacionado con el acceso a la tierra y, éste, a su vez, con las relaciones de casta.

Varios grupos en la aldea poseen ganado: los grandes terratenientes, de la casta superior Patel, tienen suficiente forraje y son dueños de importantes cantidades de ganado lechero. Los del segundo grupo, de la casta Rajput, poseen parcelas medianas y pequeñas que les permiten obtener suficiente forraje para alimentar su ganado. Los del tercer grupo, de la casta Thaku, trabajan como medieros en las tierras de la casta Patel; en estas tierras pueden obtener algo de forraje para alimentar sus animales. El cuarto grupo está formado por musulmanes que tienen pequeñas parcelas o trabajan como medieros. El quinto grupo pertenece a la casta Nai, peluqueros de oficio que prestan sus servicios a personas designadas pertenecientes a las castas superiores (Patel); estos servicios incluyen aspectos rituales religiosos, por lo cual los Nai tienen derecho a ciertas cantidades de grano y forraje de sus patrones y así pueden mantener algunas cabezas de ganado. Los demás grupos de la aldea casi no poseen ganado.

La participación de los miembros en el manejo administrativo y la toma de decisiones en la cooperativa también presenta diferencias. El analfabetismo generalizado entre las mujeres y las castas inferiores (scheduled) y el funcionamiento poco democrático de la cooperativa se identificaron como barreras a una mayor y más activa participación de la gente pobre en ella. Por ejemplo, las personas que no saben leer y escribir no pueden formar parte del comité administrativo. A estas personas les cuesta entender los procedimientos de las juntas generales y en las entrevistas dijeron que ésta era una razón para dejar de asistir a las reuniones. Algunas castas inferiores tienen que sentarse en el suelo incluso en las reuniones, en presencia de los Patel, que se dirigen a ellos con desprecio. Es obvio que este tipo de relaciones influye en la organización y en los procesos de toma de decisiones de la aldea, así como en los de todo tipo de organizaciones, entre ellas la cooperativa.

Fuente: Rawal, 1999. 
es sumamente compleja. También muestra que la forma en que opera la cooperativa guarda relación con la pobreza, un tema políticamente sensible en el caso estudiado. Varios factores impiden a muchas familias involucrarse activamente en ella. Las familias pobres que pueden lograr acceso a forraje, y por lo tanto pueden mantener animales, son excluidas de una participación activa en las decisiones de la institución. Las

\section{VI}

\section{Conclusiones}

Con este artículo se entra al debate sobre los métodos apropiados para tratar temas de desarrollo. El análisis conceptual y metodológico está dirigido a personas involucradas en estudios sobre las instituciones rurales y la reducción de la pobreza. Las instituciones rurales en las sociedades en desarrollo operan a través de relaciones complejas, tanto interpersonales como entre los hogares y a otros niveles. Por esta razón nuestro objetivo no es dictar un modelo metodológico, sino más bien proporcionar conocimientos útiles para conceptualizar y estudiar los problemas institucionales de manera flexible.

En este proyecto se integraron enfoques interdisciplinarios y se decidió utilizar el pluralismo metodológico. Los estudios de diagnóstico fueron complementados por métodos cualitativos y cuantitativos para estudiar el funcionamiento de las instituciones y su interacción con los hogares. Se examinaron los propósitos, alcances y limitaciones de cada uno de los métodos utilizados. Obviamente, son numerosos los métodos para efectuar la investigación de campo y las posibilidades analíticas para examinar los datos recolectados.

Dos tipos de conclusiones emanan del análisis de las cuestiones metodológicas vinculadas a la investigación empírica sobre las instituciones locales: algunas de orden metodológico/práctico en la realización de estudios de campo, y otras que apuntan a una mejor comprensión de las instituciones locales y su vinculación con los hogares en sus estrategias económicas, para formular recomendaciones de política a partir de la experiencia metodológica de los casos ilustrados.

Dentro del primer grupo, una de las conclusiones de la investigación es que el investigador debe combinar de manera creativa varios métodos de recopilación castas altas dominan la cooperativa y discriminan a los pobres. La discriminación hacia 'los intocables' aún se practica en Malan y las relaciones de clase son fundamentales en las relaciones de poder y en la política local, como en todas las regiones rurales de Gujarat. Es difícil imaginar procesos democráticos en contextos en que las personas son desiguales.

de información para hacer frente a la complejidad de los vínculos institucionales en las sociedades rurales. Se debe adaptar el marco de trabajo de la investigación a las condiciones locales específicas del lugar donde ella se realice.

Otra conclusión es que se requieren múltiples fases de investigación y también flexibilidad para adaptar sobre la marcha, y a criterio del equipo, los planes de la investigación de campo.

En la investigación orientada a la formulación de políticas siempre se plantea un dilema entre la restricción de tiempo y la calidad de los datos obtenidos. Esto puede resolverse hasta cierto punto con una investigación bien enfocada.

Finalmente, se vio que la formación de equipos de investigación debiera ser un componente crucial de las investigaciones. Es preciso que haya una estrecha relación de trabajo entre las agencias que ejecutan el proyecto o programa de desarrollo, por un lado, y el equipo de investigación, por otro. La formación de equipos de investigación tendría que ir en sus objetivos más allá de los objetivos directos del proyecto/programa de desarrollo, y ser considerada parte de la formación de recursos humanos a nivel local/regional/nacional.

Una segunda reflexión se refiere a lo conceptual. Para obtener y comprender información sobre instituciones en el entorno rural es preciso contar con un marco conceptual claro que evite las rigideces de las distintas propuestas teóricas y que permita captar la heterogeneidad y complejidad de los procesos institucionales. De allí que proponemos un concepto multidisciplinario que permita entender los procesos dinámicos, desde las reglas y normas establecidas hasta las prácticas organizativas de la gente. Además, subrayamos los diferentes niveles y categorías de instituciones en 
el ámbito de los estudios de caso. También destacamos la necesidad de enfocar el análisis en ciertos aspectos específicos, según los objetivos de la investigación: por ejemplo, identificar las instituciones más importantes para las actividades económicas de la gente, los procesos de inclusión y exclusión, las formas institucionales que pueden ser modificadas, mejoradas o construidas en beneficio de grupos más pobres, y la manera en que éstos puedan movilizarse para transformar las instituciones.

Queremos concluir con un breve comentario referente a ejemplos de formulación de recomendaciones de política que se pueden derivar de los estudios de casos, sobre la base del análisis conceptual y metodológico postulado en este trabajo.

El caso de San Pablo es un ejemplo interesante de una comunidad que decidió desarrollar un tipo de institución local que le puede ayudar a llevar adelante proyectos comunitarios y a negociar con agentes externos. Pero este caso también enseña la dificultad de definir objetivos institucionales que coincidan con las estrategias de los hogares individuales para generar ingresos. El análisis de la empresa forestal mostró que tanto los objetivos institucionales como los procedimientos institucionales podían ser conflictivos y problemáticos. Aunque se trataba de comunidades relativamente homogéneas desde un punto de vista cultural, la presencia de posiciones e intereses diferentes que había que considerar impedía la existencia de normas aceptadas por todos. No había nociones establecidas claras para decidir entre distribución equitativa o conservación, ni acerca de la autonomía y la dignidad. Tales nociones dependían de intereses, principios y creencias de la gente, vinculados a las fuerzas sociopolíticas y la interacción de las diferentes visiones. Por la misma razón, las posiciones eran siempre flexibles y cambiables.

Organizado en asambleas y grupos de discusión, un comité ha empezado la dura tarea de elaborar planes para la administración común y para poner por escrito las reglas y los procedimientos internos. Por medio de estas prácticas se intenta responder a los dilemas fundamentales. De la investigación puede derivarse, como recomendación de política, que sería propicio establecer mecanismos tanto de resolución de conflictos como para resolver problemas de manejo de la empresa forestal.

En el caso de la India, la investigación señaló que hay diversas instituciones que son relevantes en la vida económica de las comunidades rurales. Sin embargo, las organizaciones suelen ser dominadas por las cas- tas superiores, y diversos mecanismos adversos impiden una mayor participación de las castas pobres en las organizaciones locales. Es lo que sucede en la cooperativa lechera. En términos de recomendaciones de política, se plantearía la necesidad de encontrar los mecanismos para que, por ejemplo, las cooperativas incluyan más a las personas pobres.

Esto se relaciona también con la necesidad de que intervengan políticas públicas a un nivel más macro - por ejemplo, mediante programas de alfabetización masiva y la promoción de una mejor distribución de la tierra-, sobre todo para mejorar las condiciones de participación y negociación de la gente más pobre y discriminada.

Asimismo, en materia de recomendaciones de política, de la investigación se desprende que es fundamental, primero, apoyar a las personas en sus propios esfuerzos organizativos, aun si éstos son de naturaleza menos formal (por ejemplo, facilitando algunas relaciones con instancias burocráticas o programas gubernamentales, o dando apoyo financiero o de información). Segundo, introducir programas o proyectos dirigidos a los eslabones institucionales más débiles, reforzándolos en favor de los hogares pobres. Tercero, proporcionar mecanismos que permitan acrecentar la capacidad de acción de los propios agentes -individuales o colectivos - para que puedan movilizar y/o transformar las instituciones en beneficio de ellos mismos. Finalmente, y esto es muy importante, facilitar mecanismos de resolución de conflictos para encarar los conflictos institucionales que puedan afectar a los hogares pobres en su acceso a los recursos.

$\mathrm{Al}$ tener como objetivo ciertas instituciones u organizaciones específicas dentro de programas y proyectos de desarrollo, es importante una aproximación abierta y flexible que tome en cuenta las iniciativas locales, y que además contemple los grupos e intereses diversos, dando lugar a espacios amplios de negociación abierta e inclusiva. En esta perspectiva es importante definir las áreas, formas y niveles en que puede llevarse a cabo la intervención, ya que las instituciones se componen de diversos elementos a distintos niveles que van desde la comunidad y la aldea hasta las instancias gubernamentales y la legislación nacional. Así, las acciones a nivel local pueden articularse con programas públicos amplios, como programas educativos masivos, que en última instancia pueden ser medios para el "empoderamiento" de los grupos más pobres y una estrategia general para ampliar su participación en los ámbitos institucionales y organizativos locales. 
Alaasutari, P. (1995): Researching Culture: Qualitative Methods and Cultural Studies, Sage, Londres.

Appadurai, A. (1997): Modernity at Large: Cultural Dimensions of Globalization. Minnesota, University of Minnesota Press.

Appendini, K., R. García y B. de la Tejera (2001): Institutional flexibility in a transnational community: managing collective natural resources, en A. Zoomers (ed.), Land and Sustainable Livelihood, Amsterdam, Países Bajos, Royal Tropical Institute.

Bebbington, A. y J. Ferrington (eds.) (1993): Reluctant Partners, Non Governmental Organizations, the State and Sustainable Agricultural Development, Londres, Routledge.

CRIM/UNAM/CRUCO/UACH (Centro Regional de Investigaciones Multidisciplinarias/Universidad Nacional Autónoma de México/Centro Regional Universitario Centro Occidente/Universidad Autónoma de Chapingo) (1999): Estrategias de ingreso en los hogares rurales para alivio de la pobreza e interacciones con las instituciones locales: Caso México, documento inédito, Cuernavaca, Morelos, México.

Crowley, E. y K. Appendini (1998): Rural poverty: population dynamics, local institutions and access to resources, documento presentado al taller temático sobre Población, Pobreza y el Medio Ambiente, Roma, Organización de las Naciones Unidas para la Alimentación y la Agricultura (FAO)/Organización Internacional del Trabajo (OIT)/Fondo de las Naciones Unidas para actividades de población (UNFPA), octubre.

Curtis, D. (1991): Beyond government: Organizations for Common Benefit, MacMillan, Londres.

Esman, M. y N. Uphoff (1984): Local Organisations: Intermediaries in Rural Development, Ithaca, Nueva York, Cornell University Press.

Harris, M. (1988): On Charities and NGOs, en R. Poulton y M. Harris (eds), Putting People First: Voluntary Organisations and Third World Organisations, MacMillan, Londres.

IIM (Indian Institute of Management) (1999): Rural Household Income Strategies for Poverty Alleviation and Interactions with the Local Institutional Environment: A Study of India, Ahmedabad, India, IIM.

Korten, D., ed. (1987): Community Management: Asian Experiences and Perspectives, West Harford, Connecticut, Kumarian Press.

Leach, M., R. Mearns y R., Scoones (1997): Institutions, consensus and conflict; implications for policy and practice, IDS Bulletin, Vol. 28, N 4, Sussex, Reino Unido, Universidad de Sussex, Instituto de Estudios para el Desarrollo.

Long, N. (1990): From paradigm lost to paradigm regained? The case for an actor-oriented sociology of development, European Review of Latin American and Caribbean Studies, $\mathrm{N}^{\circ} 49$, Amsterdam, Países Bajos, Centro Universitario de Estudios y Documentación Latinoamericanos (CEDLA).

Mikkelsen, B. (1995): Methods for Development Work and Research: a Guide for Practitioners, Londres, Sage Publishers.

Mitchell, C. (1983): Case and situation analysis. Sociological Review, $\mathrm{N}^{\circ}$ 31, vol. 2, Oxford, Reino Unido, Basil Blackwell.

Mosse, D. (1994): Authority, gender and knowledge: Theoretical reflections on the practice of participatory rural apppraisal, Development and Change, vol. $25, \mathrm{~N}^{\circ} 3$, Londres, Instituto de Estudios Sociales (ISS).
(1998): Process-oriented approaches to development practice and social research, en D. Mosse, J. Farrington y A. Rew (eds.), Development as Process: Concepts and Methods for Working with Complexity, Nueva York, Routledge.

North, D. (1990): Institutions, Institutional Change and Economic Performance, Cambridge, Massachusetts, Cambridge University Press.

Nuijten, M. (1998): In the name of the land; organisation, transnationalism and the culture of the State in a Mexican ejido, Wageningen, Wageningen Agricultural University, tesis de doctorado.

Ostrom, E. (1995): Governing the Commons: The Evolution of Institutions for Collective Action, Cambridge, Massachusetts, Cambridge University Press.

Poulton, R. y M. Harris, eds. (1988): Putting People First: Voluntary Organisations and Third World Organisations, MacMillan, Londres.

Rawal, V. (1999): Participation of the poor in dairy cooperatives in Gujarat: Studying exclusion in rural institutions, artículo de antecedente al proyecto Rural Household Income Strategies for poverty alleviation and interactions with the local institutional environment, Roma, Organización de las Naciones Unidas para la Alimentación y la Agricultura (FAO), División de Desarrollo Rural, inédito.

Reed, M. (1992): The Sociology of Organisations; Themes, Perspectives and Prospects, Nueva York y Londres, Harvester Wheatsheaf.

Richards, P. (1995): Participatory Rural Appraisal: A Quick and Dirty Critique, Notes on Participatory Learning and Action, $\mathrm{N}^{\circ} 24$, Londres, Instituto Internacional para el Medio Ambiente y el Desarrollo.

Scott, W. (1995): Institutions and Organisations. Thousand Oaks, California.

Silverman, D. (1993): Interpreting Qualitative Data: Methods for Analysing Talk, Text, and Interaction, Sage, Londres.

Starr, J. y J. Collier (1989): History and Power in the Study of Law: New Directions in Legal Anthropology, Ithaca, Cornell University Press.

Strauss, A. (1987): Qualitative Analysis for Social Scientists, Cambridge, Massachusetts, Cambridge University Press.

UEM (Universidade Eduardo Mondale) (1999): Estratégias de geração de renda das familias rurais e suas interaccões com o ambiente institucional local, Maputo, Mozambique, UEM, Faculdade de Agronomia e Engenharia Florestal.

Uphoff, N. (1986): Local Institutional Development: An Analytical Sourcebook with Cases, West Harford, Connecticut, Kumarian Press.

Walton, J. (1992): Making the theoretical case, en H. Becker y C. Ragin (eds.), What is a Case? Exploring the Foundations of Social Inquiry, Cambridge, Massachusetts, Cambridge University Press.

Wolf, E. (1990): Facing power: Old insights, new question, American Anthropologist, vol. 92, $\mathrm{N}^{\circ}$ 3, Washington, American Anthropological Association. 